\title{
Adherence to the National Guidelines on the management of bronchial asthma: a cross-sectional study in Medical Clinics in Teaching Hospital, Karapitiya.
}

\author{
Fernando GNN, De Silva KHPUD \\ Teaching Hospital Karapitiya, Galle \\ Corresponding author:Dr.GNNFernando(nilukshignn@yahoo.com)
}

\begin{abstract}
Introduction: In persistent asthma, the regular use of inhaled corticosteroids (ICS) on daily basis is the mainstay of therapy. As little is known about the use of ICS in the local set up, this study was planned to identify how the ICS are used, compliance of patients and factors that limit the correct use of ICS.

Objectives: To describe the adherence to the guidelines; in prescribing and dose titration and to assess the correct technique of using inhaler device. Also to describe patient factors that limit adherence to guidelines.

Methods: This is a descriptive cross-sectional study involving 106 Physician-diagnosed bronchial asthmatics attending medical clinics in Teaching Hospital, Karapitiya.

Results: Patients were between the ages of $12-93$ years. $67 \%$ were between $41-70$ years. $67.9 \%$ patients were prescribed appropriate optimal treatment according to the guidelines. $67 \%$ of patients showed a good compliance to the prescribed medications. The commonest reasons for poor compliance were unaffordability of drugs $(56.75 \%)$ and improvement of symptoms as felt by the patients $(18.9 \%)$. Compliance declined with the duration of follow up. $64.2 \%$ were following the correct inhaler technique. The most critical step in the inhaler technique was the 'breath holding for 10 seconds' (only $41.3 \%$ adhered to this). Males were significantly (chisquare test; $p=0.01$ ) better in practicing the proper inhaler technique as compared to females.

Conclusions: Majority of patients need ICS as the primary treatment with some requiring long acting bronchodilators. Programs to improve the knowledge of doctors would be beneficial to improve the adherence to the guidelines. Supplying ICS free of charge by the hospital, at least in the initial years, and continuous health education would improve the compliance to ICS. The inhaler technique should be checked at least in nonresponders. When checking the inhaler technique special attention should be paid to the ability of holding breath after inhalation and females are the most vulnerable group. Changing over to metered dose inhaler with a spacer device should not be delayed in repeated failures of dry powder inhalers.
\end{abstract}

\section{Introduction}

Incidence of bronchial asthma has risen worldwide over past decades (1). Due to the introduction of long term inhaled corticosteroids (ICS) many asthma sufferers have less exacerbations and less disturb-ances to their daily activities, thus improving the quality of their lives (2). In spite of ICS use, some patients continue to have frequent exacerbations and poor quality of life. This study was planned to study how ICS are being practiced, treatment compliance and factors that limit the correct use of ICS in medical clinics in Teaching Hospital Karapitiya.

\section{Clinical standards}

The clinical standards of this study was defined by the recommendation given in the "SLMA guidelines on the management of Asthma"

Recommendations in these guidelines address following areas.

(1) Assessment of severity of asthma according to the guidelines. (Pg 23 SLMA Guidelines)

(2) Assessment of drug prescribing and correct dose titration according to the guideline.(Pg 37 SLMA Guidelines)

(3) Technique of use of inhale devices. (Pg 45, SLMA Guidelines). 


\section{Objectives of the study included the following}

(1) To describe the adherence to the guidelines; in prescribing and dose titration.

(2) To assess the correct technique of using inhaler device.

(3) To describe patient factors that limit the adherence to the guidelines.

\section{Methods}

\section{Design and set up}

This was a descriptive cross-sectional study involving Physician-diagnosed bronchial asthmatics attending medical clinics in Teaching Hospital, Karapitiya.

\section{Sampling}

We included all consecutive asthmatics attending morning medical clinics at Teaching Hospital Karapitiya during the month of September 2009, until the required sample size was achieved. Patients with other co-morbid diseases contributing to dyspnoea were excluded. Sample size of 106 was determined based on the formula given by Lwanga and Lemeshow $(3,4)$.

\section{Data collection}

The current severity of disease was assessed by the first investigator. The current prescription was compared with the standards given in the guidelines, (Clinical standards 1 and 2) as the current drug regimen reflects the combination of correct drug prescription and appropriate dose titration. In addition clinical notes were reviewed to trace written records of previous prescriptions and dose titration. Patients were asked to demonstrate the inhaler technique and the observations were compared with the standards (Clinical standard 3). Various patientrelated factors that limit the adherence to the guidelines were gathered by interviewing the patient and reviewing clinical notes.

An interviewer-administered structured question-naire was used to collect patients' information. Informed consent was obtained from each patient before the interview. Each patient's demographic profile and other relevant data to achieve the above mentioned objectives were also recorded.

\section{Data analysis}

Data were entered and analyzed using SPSS and Excel software. Statistical analysis was performed using Chi square test. $\mathrm{P}$ value of $<0.05$ was considered statistically significant.

\section{Results}

\section{Characteristics of the patients}

There were 106 patients (37 male) in the study sample. Table 1 shows the demographic characteristics of the patients. Patients were between the ages of 12-93 years and 71(67\%) were between 41-70 years. Male to female ratio was $1: 1.9 .79$ (74.5\%) patients have had secondary school education. Eighty two (77.3\%) subjects were partially skilled or unskilled workers receiving a lower income while 41 (38.7\%) were engaged in dust-related occupations.

Table 1: Demographic characteristics of patients

\begin{tabular}{ll}
\hline Variable & $\begin{array}{c}\text { Number } \\
\text { (Percentage) }\end{array}$ \\
\hline Age group & $9(9 \%)$ \\
$<31$ & $6(5 \%)$ \\
$31-40$ & $22(21 \%)$ \\
$41-50$ & $17(16 \%)$ \\
$51-60$ & $32(30 \%)$ \\
$61-70$ & $16(15 \%)$ \\
$71-80$ & $4(4 \%)$ \\
$>80$ & \\
Level of education & \\
Never gone to school & $6(5.5 \%)$ \\
Primary (gr 1-5) & $21(19.8 \%)$ \\
Secondary (gr 6-9) & $37(34.9 \%)$ \\
O/L pass & $33(31.1 \%)$ \\
A/L pass & $9(8.5 \%)$ \\
& \\
Social class & \\
Class I(leading professions) & $0(0 \%)$ \\
Class II(lesser professions) & $11(10.4 \%)$ \\
Class III(skilled workers) & $13(12.3 \%)$ \\
Class IV(partial skilled workers) & $42(39.6 \%)$ \\
Class V(unskilled workers) & $40(37.7 \%)$ \\
& \\
Smoking history & \\
Never & $74(69.8 \%)$ \\
Past smoker & $19(17.9 \%)$ \\
Current active smoker & $2(1.9 \%)$ \\
Passive smoker & $11(10.4 \%)$ \\
\hline
\end{tabular}


The majority of the patients were below the Grade III according to the asthma severity classification. (Table 2).

Table 2: Distribution of the severity (Grade) of asthma

\begin{tabular}{|c|c|}
\hline Severity Grade & Number (Percentage) \\
\hline$<$ I & $8 \quad(7.5 \%)$ \\
\hline I & $26(24.5 \%)$ \\
\hline I-II & $10(9.4 \%)$ \\
\hline II & $29(27.4 \%)$ \\
\hline II - III & $21(19.8 \%)$ \\
\hline III & $5 \quad(4.7 \%)$ \\
\hline III-IV & $4 \quad(3.8 \%)$ \\
\hline IV & $3 \quad(2.8 \%)$ \\
\hline
\end{tabular}

Of 106 asthmatics analyzed, 105 were on inhalers. ICS were prescribed to $91(85.8 \%)$ patients while ICS and long acting beta agonists (LABA) combination were prescribed to $13(12.3 \%)$ patients. LABAalone was prescribed to one patient $(0.9 \%)$.

\section{Adherence to guidelines on prescription}

According to the guidelines, 72 (67.9\%) patients were prescribed an appropriate optimal treatment while $18(17 \%)$ were under-treated and $16(15.1 \%)$ were over-treated.

\section{Compliance to ICS and patient factors that limit compliance}

Seventy one $(67 \%)$ patients had good compliance to the prescribed medications (following the prescription at least $90 \%$ days in a month) while 31 $(29.2 \%)$ showed poor compliance (following the prescription 50\%-90\% days in a month) and 4 patients $(3.8 \%)$ had very poor compliance (following the prescription $<50 \%$ days in a month).

Reasons for the poor compliance were assessed in 31 subjects who were not fully compliant with the prescribed medication. Twenty one $(56.8 \%)$ said the treatment was not affordable while two subjects experienced side effects. Six were not compliant due to their busy work schedule and seven felt clinically well and stopped treatment on their own.
Of 71 subjects who had good compliance, gender difference $($ male/female $=27 / 44)$ was not statistically different (Chi square test; $p=0.3$ ). Even though there was no association between the duration of follow up and the compliance to ICS (chi square test; $p=0.8$ ), compliance declined with the duration of follow up. (Table 3).

Table 3: Percentage of patients with good compliance (following the prescription at least $\mathbf{9 0 \%}$ days of a month) to ICS according to the duration of follow up

\begin{tabular}{cc}
\hline $\begin{array}{l}\text { Duration of } \\
\text { follow up }\end{array}$ & $\begin{array}{c}\text { Percentage of patients } \\
\text { with good compliance }\end{array}$ \\
\hline$<1$ year & $71.4 \%$ \\
1-3 years & $69.4 \%$ \\
$>4$ years & $65.6 \%$ \\
$>10$ years & $54.4 \%$ \\
\hline
\end{tabular}

The percentages of subjects with good compliance (following the prescription at least $90 \%$ of the month) did not vary according to the educational level (chi square test; $\mathrm{p}=0.5$ ) (Table 4).

Table 4: Percentage of patients with good compliance according to the educational level

\begin{tabular}{cc}
\hline Educational level & $\begin{array}{c}\text { Percentage of patients } \\
\text { with good compliance }\end{array}$ \\
\hline Never & $50 \%$ \\
$<$ Grade 5 & $81 \%$ \\
Grade $5-9$ & $59.5 \%$ \\
O/L pass & $69.7 \%$ \\
A/L pass & $66.7 \%$ \\
\hline
\end{tabular}

\section{Assessment of inhaler technique}

Of 106 patients, 101(96.2\%) were using dry powder inhalers while $3(2.9 \%)$ were using metered dose inhaler (MDI) with a spacer and one $(0.9 \%)$ was using MDI without a spacer device. Furthermore, 68 $(64.2 \%)$ were following the correct inhaler technique. Table 5 shows the distribution of patients who were correctly performing each step. 
Table 5: Percentage of patients demonstrating correct technique in each step of the inhaler technique

\begin{tabular}{llll}
\hline Step of inhaler technique & Total & Male & Female \\
\hline Proper loading of capsule & $90.5 \%$ & $89.2 \%$ & $91.2 \%$ \\
Good deep inspiration & $89.6 \%$ & $97.3 \%$ & $85.3 \%$ \\
Keep closed mouth after inhalation & $80 \%$ & $81.1 \%$ & $79.4 \%$ \\
Keep breath hold & $41.3 \%$ & $62.2 \%$ & $29.9 \%$ \\
Do repeat cycles & $90.5 \%$ & $89.2 \%$ & $91.2 \%$ \\
Do gargling & $85.3 \%$ & $83.3 \%$ & $86.4 \%$ \\
\hline
\end{tabular}

Males were significantly (chi-square test; $p=0.01$ ) better in practicing the proper inhaler technique when compared to females. (Table5)

\section{Adverse effects experienced by patients}

Of all patients using ICS, 79 (73.8\%) did not have any side effects related to the drug. The most common side effect was dry cough (12.1\%). Ten $(9.3 \%)$ experienced sore throat. Oral infections and voice change, well known side effects of ICS, were seen in only one and two subjects, respectively.

\section{Patient's knowledge on cleaning and renewal of device}

Of all using inhaler devices, 94 (88.7\%) cleaned the inhaler device properly (wash with water at least once a week) while $2(1.9 \%)$ patients washed the device infrequently. $7(6.6 \%)$ wiped the inhaler device with a cloth and $3(2.8 \%)$ did not clean the device at all. Majority (51\%) changed the device within 6 months to 2 years.(Table6)

Table 6: Frequency of changing a new inhaler device

\begin{tabular}{ccc}
\hline Frequency of changing & \multicolumn{2}{c}{ Number (Percentage) } \\
\hline Within 6 months & 5 & $(4.7 \%)$ \\
6-12 months & $32(30.2 \%)$ \\
12-24 months & $22(20.8 \%)$ \\
$>24$ months & $16(15.1 \%)$ \\
$>5$ years /never & $17(16 \%)$ \\
Not applicable (clinic & \\
attending <6months) & $14(13.2 \%)$ \\
\hline
\end{tabular}

\section{Discussion}

The recorded annual asthma admissions and deaths in the state sector in Sri Lanka have shown a steady increase over the past decade with 180,000 admissions and 800 deaths in 2002 (5). These figures indicate that asthma is a major health problem in Sri Lanka. Even though the primary therapy for persistent asthma is the regular use of ICS on a daily basis, little is known about compliance, adherence to guidelines, reasons for poor compliance and the correct practice of inhaler devices in Sri Lanka.

This study was done in medical clinics of Teaching Hospital, Karapitiya on 106 Physician- diagnosed patients with bronchial asthma. $67 \%$ were between 41 to 70 years with a male to female ratio of $1: 1.9$ (Table 1). In general, asthma is more prevalent in children and young adults, but the majority of the sample was in middle and elderly age groups, partly due to sampling in medical clinics and partly due to the population structure of Sri Lanka. On the other hand the study did not reveal the age of onset of the disease. The number of females was almost double the number of males which can be partly explained by the sex ratio in Sri Lanka and by the higher health seeking behavior of females when compared to males.

As $56.7 \%$ were in Grades II and III of asthma severity classification and needed ICS as the primary treatment supplemented by adding LABA, steps should be taken to avoid overtreatment of less severe cases as still a quarter $(24.5 \%)$ of the patients did not require commencement of ICS due to the intermittent nature of their disease (Table 2). 
Adherence to the guidelines on prescription and dose titration occurred in only $67.9 \%$ which shows the need for further awareness programs to improve the knowledge of doctors. $67 \%$ of patients had good compliance to ICS. The commonest reason for poor compliance was unaffordability $(56.75 \%)$ of drugs as they are not always available at clinics. Providing ICS free of charge by the state, at least in the initial years, will be more beneficial. The second most common reason for poor compliance was improvement of symptoms as felt by the patients (18.9\%). This highlights the importance of continued health education with special emphasis on the need to continue medicine, even when the patient feels well. There was no significant association between compliance and gender or educational level, but $81 \%$ of the patients educated at primary level had been well compliant in contrast to patients educated to secondary level; probably they may have taken their own decision to stop drugs (Table 4). The poorest compliance (50\%) was within the uneducated group. This denotes the importance of focusing the attention first to uneducated and then to secondary educated patients with regard to poor compliance. Also the compliance drops with the duration of follow up; more attention should be given to patients with prolonged follow up (Table 3).

Out of all, $68(64.2 \%)$ were following the correct inhaler technique. The poorest step in the technique was the 'breath holding for 10 seconds' (only $41.3 \%$ adhered to this). This shows the importance of checking the inhaler technique at least in nonresponders; specially the ability to hold breath after deep inhalation should be observed. Special attention should be given to females as their inhaler technique is significantly worse when compared to males. Males were good in 'deep inspiration', 'keeping the mouth closed after inhalation' and particularly the 'breath holding' compared to females (Table 5). For patients with continued symptoms while on dry powder inhalation and despite the proper instructions, a MDI with a spacer device should be commenced early. The use of MDI with spacer was very low (2.9\%) and probably it reflects the hesitancy of doctors to prescribe them possibly due to cost factors.

In the entire sample, $73.8 \%$ did not experience any adverse effects following the use of ICS. The commonest adverse effect experienced by the patients was dry cough (12.1\%) and sore throat $(9.3 \%)$. These patients should be advised on gargling of throat properly. For persistent sufferers MDI can be recommended. $88.7 \%$ of patients knew the proper method of cleaning the inhaler device. Only $30.2 \%$ of patients changed the new inhaler device within 612 months (compatible with guidelines). But about half of the patients were (51\%) changing the device at the end of 2 years (Table 6 ).

\section{Limitations}

This study included a group of bronchial asthmatics diagnosed by physicians. As the diagnosis of asthma is made on clinical grounds, an overlap with other diseases which share the same symptomatology is unavoidable. Although we took extra precautions to exclude patients with fixed airway obstruction, some patients with chronic obstructive airway disease may have been included in the study. This is one of the limitations of this analysis. Furthermore, the compliance was assessed on the information given by patents and there was no way to check the accuracy of this information.

\section{Conclusions}

Majority of patients need ICS with LABD as the primary treatment for a better control of asthma. Steps should be taken to avoid overtreatment of less severe cases as still a quarter $(24.5 \%)$ of the patients did not require commencement of ICS. Further, awareness programs to improve the knowledge of doctors would be beneficial to improve the adherence to the guidelines. Supply of ICS free of charge by the state and continuous health education of patients with special emphasis on the need to continue medicine, even when the patient feels well, would improve the compliance to ICS.

The inhaler technique should be checked at least in non-responders; specially the ability of 'breath holding' after deep inhalation and special attention should be given to females as their inhaler technique is significantly worse compared to males. Changing over to MDI with a spacer device should not be delayed in repeated failures to dry powder inhalers. 


\section{Acknowledgment}

We would like to acknowledge all Consultants who gave permission to include their patients in the study, health care professionals, administrative staff at Teaching Hospital Karapitiya and all the patients who contributed to this study. Wish to thank Dr. A. Kasthurirathne and Dr. N. Perera, Department of Community Medicine, Faculty of Medicine, University of Kelaniya for advising with statistical analysis.

\section{References}

1. 2008 University of Maryland medical center. Asthma in Adults. www.umm.edu.Asthma in Adult. (Accessed on $6^{\text {th }}$ ofFeb 2010)

2. Asthma management hand book 2006.National Asthma Council Australia. http://www.NationalAstma.org.au (last updated on 2009 and last accessed on $10^{\text {th }}$ of Feb 2010)

3. Lwanga S.K. Lemeshow S. Sample size determination in health studies; a practical manual. Geneva, WHO. pp 25.

4. GM Cochrane, CR horn. Management of Bronchial Asthma in Community; Problems of Compliance with Treatment. Quarterly Journal of Medicine 1991; 81(294): 797-798.

5. SLMA Guidelines on Management of Asthma, 2005, $3^{\text {rd }}$ Edition Produced by the Sri Lanka Medical Association in 2005. 